\title{
Effect of grade on disease-free survival and overall survival in FIGO stage I adenocarcinoma of the endometrium
}

\author{
Jean F. Delaloye (M.D.) $)^{\mathrm{a}}$, Sandro Pampallona (D.Sc.) $)^{\mathrm{b}}$, Philippe A. Coucke (M.D.), \\ Alexandre Megalo (M.D.) $)^{\mathrm{a}}$, Pierre De Grandi (M.D. Prof.) ${ }^{\mathrm{a}}$ \\ ${ }^{a}$ Département de Gynécologie-Obstétrique, Centre Hospitalier Universitaire Vaudois, 1011 Lausanne, Switzerland \\ ${ }^{\mathrm{b}}$ forMed, 1983 Evolène, Switzerland \\ ${ }^{\mathrm{c}}$ Service de Radio-Oncologie, Centre Hospitalier Universitaire Vaudois, 1011 Lausanne, Switzerland
}

Received 30 July 1998; accepted 20 April 1999

\begin{abstract}
Objective: To analyse the effect of differentiation on disease-free survival (DFS) and overall survival (OS) in patients with stage I adenocarcinoma of the endometrium. Patients and methods: From 1979 to 1995, 350 patients with FIGO stage IA-IC with well (G1), moderately (G2) or poorly (G3) differentiated tumors were treated with surgery and high dose-rate brachytherapy with or without external radiation. Median age was 65 years (39-86 years). Results: The 5-year DFS was $88 \pm 3 \%$ for the G1 tumors, $77 \pm 4 \%$ for the G2 tumors, and $67 \pm 7 \%$ for the $\mathrm{G} 3$ tumors $(P=0.0049)$. With regard to the events contributing to DFS, the 5-year cumulative percentage of local relapse was $4.6 \%$ for the G1 tumors, $9.0 \%$ for the G2 tumors, and $4.6 \%(P=0.027)$ for the G3 tumors. Cumulative percentage of metastasis was $1.4,6.3$ and $7.2 \%(P<0.001)$, respectively, whereas percentages of death were 6.0, 7.9 and 20.7\% $(P<0.001)$. The 5-year OS was $91 \pm 3,83 \pm 4$ and $76 \pm 7 \%$, respectively $(P=0.0018)$. In terms of multivariate hazard ratios $(\mathrm{HR})$, the relative differences between the three differentiation groups correspond to an increase of $77 \%$ of the risk of occurrence of either of the three events considered for the DFS $(\mathrm{HR}=1.77,95 \% \mathrm{Cl}[0.94-3.33]),(P=0.078)$ for the $\mathrm{G} 2$ tumors and of $163 \%(\mathrm{HR}=2.63,95 \% \mathrm{Cl}[1.27-5.43]),(P=0.009)$ for the G3 tumors with respect to the G1 tumors. The estimated relative hazards for OS are, respectively, in line with those for DFS: HR=1.51 $(P=0.282)$ for the $\mathrm{G} 2$ tumors; and $\mathrm{HR}=3.37(P=0.003)$ for the $\mathrm{G} 3$ tumors. Conclusion: Patients with grade 1 tumors are those least exposed to either local relapse, metastasis, or death. In contrast patients with grade 2 tumors seem to be at higher risk of metastasis, whereas patients with grade 3 tumors appear at higher risk of death. Since we have looked at the first of three competing events (local relapse, metastasis and death), this suggests that patients with grade 3 tumors probably progress to death so fast that local relapse, if any, cannot be observed. (c) 2000 Elsevier Science Ireland Ltd. All rights reserved.
\end{abstract}

\section{Introduction}

Cell differentiation has been shown to be one of the most important prognostic factors in carcinoma of the endometrium [1-4]. In this retrospective analysis we aimed to investigate the effect of grade on disease control in FIGO stage I patients [5], who were treated with a combination of surgery and postoperative radiotherapy

*Corresponding author. Tel.: +41-21-3143269; fax: +41-21-3143263. based on the myometrial invasion only, independently of cell differentiation.

\section{Patients and methods}

\subsection{Patient population}

From April 1979 to December 1995, 350 patients with primary FIGO stage IA-IC pure adenocarcinoma of the endometrium were treated in the Centre Hospitalier Uni- 
Table 1

Patients characteristics

\begin{tabular}{lc}
\hline Prognostic factors & Total $(\%)$ \\
\hline Number of patients & 350 \\
Median age (years) & 65 \\
FIGO stage: & \\
IA & $67(19)$ \\
IB & $186(53)$ \\
IC & $97(28)$ \\
Differentiation: & \\
G1 & $166(47.5)$ \\
G2 & $123(35)$ \\
G3 & $61(17.5)$ \\
\hline
\end{tabular}

versitaire Vaudois, Lausanne, Switzerland. Patients characteristics are summarized in Table 1 . The median age was 65 years (range 36-86 years). The tumors were classified in three grades: well (G1), moderately (G2) or poorly (G3), according to the WHO cellular differentiation [5]. Slides were reviewed by two pathologists.

\subsection{Treatment}

All patients underwent total extrafascial hysterectomy and bilateral salpingo-oophorectomy. Postoperative treatment strategy was planned on the pathology report of the myometrial invasion only, independently of the cellular differentiation. Peritoneal washing was not routinely obtained and lymph nodes dissection was very uncommon. All 350 patients received vaginal vault irradiation. In addition 56/186 patients with stage IB greater than $30 \%$ myometrial infiltration, and all 97 patients with stage IC received external irradiation.

\subsection{Radiation therapy}

Four weeks after surgery, external irradiation was given on the pelvic volume with a linear accelerator $(6-18 \mathrm{MeV})$ for a total dose of $4500 \mathrm{cGy}$ fractionated at a daily dose of 180-200 cGy [6-8]. The reference point for dose prescription was at the intersection of the four beams. The upper limit of the pelvic volume was defined by a line passing through the L5-S1 interspace. The lateral borders were located $1 \mathrm{~cm}$ beyond the bony pelvis. The inferior border covered two-thirds of the vagina.

Brachytherapy delivered a boost of 45 Gy at the surface of the vaginal cuff over 2 weeks (three fractions) with a high dose-rate $\left({ }^{137} \mathrm{Cs}\right)$ remote afterloading system.

\subsection{Statistical methods}

The primary endpoint of this retrospective study was disease-free survival (DFS), which was defined as time from surgery to the first of the three competing events: local recurrence, metastasis or death, whichever occurred first. The secondary end-point was overall survival, defined as time from surgery to death.

Statistical analyses were carried out on the software package Stata [9]. Survival percentages over time have been calculated according to the Kaplan-Meier method [10], and their corresponding standard errors (SEs) with Greenwood's formula [11]. For the univariate analysis the $P$ values from the log-rank test are reported [12]. Estimated hazard ratios (HR) for DFS and OS, their 95\% confidence intervals $(95 \% \mathrm{Cl})$ and $P$ values were calculated with a multivariate proportional hazard-regression model [13]. Appropriate binary indicators have been defined to identify the categories of the following variables: age (50, 51-60, 61-70, 71), FIGO stage IA-IC, grades $1-3$. Variables describing duration and intensity of radiation therapy have not been considered given the homogeneity of treatment delivered. HRs quoted in the Section 3 are from a model including all above variables. Values of HR greater than unity indicate increased rates of any of the events contributing to death (or of death for OS) with respect to the chosen reference category (for which $\mathrm{HR}=1$ by definition). The probability that local progression or metastasis or death occurs before any specified time has been estimated by means of cumulative incidence functions [14]. For the sake of interpretation we point out that one minus the sum of such probabilities (local relapse, metastasis, death), at any given point in time, corresponds to the probability of being alive and disease-free as obtained from the Kaplan-Meier estimate of the DFS. Appropriate statistical methods have been used for comparing cumulative incidences allowing for stratification factors [15]. All probability values are for two-sided tests. For the purpose of the analyses, observations have been censored at 5 years in order to limit the effect of competing causes of mortality and also to avoid having only patients registered during the earlier periods contribute to the right tail of the curves.

\section{Results}

\subsection{Association among patient characteristics}

Age was associated with tumor stage. Patients with FIGO stage IA were younger (63 years) than those with stage IB (65 years) or stage IC (67 years) $(P=0.0036)$. No other association among prognostic factor was found.

\subsection{Disease-free survival analysis}

The median follow-up was in excess of 7 years. As explained in Section 2, DFS and OS have been censored at 5 years. A total of 55 events have been observed by the fifth year. The 5 -year DFS was $80 \pm 8 \%$. When considering sub-groups, the 5-year DFS was $88 \pm 3 \%$ for the G1 group, 
$77 \pm 4 \%$ for the G2 group, and $67.7 \%$ for the G3 group $(P=0.0049)$. In terms of multivariate hazard ratio $(\mathrm{HR})$, the relative difference between the three groups corresponds to an increase of $77 \%$ of the risk of occurrence of either of the three events considered (local relapse, metastasis and death) $(\mathrm{HR}=1.77,95 \% \mathrm{Cl}[0.94-3.33])(P=$ $0.078)$ for the $\mathrm{G} 2$ group, and of $163 \%(\mathrm{HR}=2.63,95 \% \mathrm{Cl}$ [1.27-5.43] $)(P=0.009)$ for the G3 group with respect to G1 group. These and additional multivariate results are displayed in Table 2. Local relapse was defined as vaginal and/or latero-pelvic recurrence.

\subsection{Cumulative incidence analysis}

In order to better investigate the effect of grade on the pattern of occurrence of the events contributing to DFS (local relapse, metastasis and death), we have estimated the cumulative incidence of each of these competing events individually. The results are summarized in Table 3. With regard to cell differentiation, the 5-year cumulative percentage of local relapses was $4.6 \%$ for grade $1,9.0 \%$ for grade 2 , and $4.6 \%$ for grade $3(P=0.027)$. Similarly, such percentages were 1.4, 6.3 and $7.2 \%(P<0.001)$ for metastasis, and 6.0, 7.9 and $20.7 \%(P<0.001)$ for deaths. The cumulative incidence curves are displayed in Figs. 1-3. Fig. 1 for local recurrence shows a rapid increase of the curve of the grade 2 tumors during the first year after treatment, and a slower increase and subsequent leveling off of the curves in the G1 and G3 groups by the third year. Fig. 2 for distant metastasis shows a slow increase and subsequent levelling off of the curves in the G2 and G3 groups at three years. As concern deaths, Fig. 3 suggests that they continue to accumulate over time in all differentiation groups, but again much faster for G3. The 5-year percentage of deaths is almost triple compared to what has been observed in the G1 and G2 groups.
Table 3

Cumulative incidences (\%) of three events contributing to DFS ${ }^{\mathrm{a}}$

\begin{tabular}{llcc}
\hline Grade & Local relapse & Metastasis & Death \\
\hline 1 & 4.6 & 1.4 & 6.0 \\
2 & 9.0 & 6.3 & 7.9 \\
3 & 4.6 & 7.2 & 20.7 \\
$P$ value & 0.027 & $<0.001$ & $<0.001$ \\
\hline
\end{tabular}

${ }^{a}$ Standard errors are in all cases less than $3 \%$. The reported $P$ value are from an adjusted test, stratifed by FIGO stage.

\subsection{Overall survival analysis}

The 5-year overall survival was $84.8 \pm 2 \%$. When considering sub-groups, the 5-year OS was $91 \pm 3 \%$ for the G1 group, $83 \pm 4 \%$ for the $\mathrm{G} 2$ group, and $67.7 \%$ for the $\mathrm{G} 3$ group $(P=0.0018)$. In terms of multivariate hazard ratio (HR), the relative difference between the three groups corresponds to a non-significant increase of $51 \%$ of the risk of death for the $\mathrm{G} 2$ group $(\mathrm{HR}=1.51,95 \% \mathrm{Cl}[0.71-$ 3.19]) $(P=0.282)$, and of $237 \%$ for the G3 group ( $\mathrm{HR}=$ $3.37,95 \% \mathrm{Cl}[1.52-7.44])(P=0.003)$ with respect to the G1 group (Table 2).

\section{Discussion}

Our study confirms the prognostic importance of cell differentiation on disease-free survival (DFS) and overall survival (OS) of adenocarcinoma of the endometrium. The relative shapes of the cumulative incidence curves suggest that the strong reduction in DFS beyond the first year after treatment for patients with G3 differentiation can be attributed to death. As in other series, well-differentiated (G1) tumors were easily locally controlled, resulting in a longer survival. In contrast, moderately (G2) and poorly (G3) differentiated tumors were characterised by metastases and death, respectively [1,16-22]. G2 patients pro-

Table 2

Cox's analysis fitted to the overall survival (OS) and disease-free survival (DFS)

\begin{tabular}{|c|c|c|c|c|}
\hline & $\begin{array}{l}\text { OS } \\
\text { HR (95\% CI) }\end{array}$ & $P$ value & $\begin{array}{l}\text { DFS } \\
\text { HR }(95 \% \mathrm{CI})\end{array}$ & $P$ value \\
\hline \multicolumn{5}{|c|}{ Age (years): } \\
\hline 50 & Reference category & & Reference category & \\
\hline $51-60$ & $1.85(0.21-16.85)$ & 0.577 & $0.86(0.16-4.77)$ & 0.871 \\
\hline $61-70$ & $2.51(0.33-19.05)$ & 0.371 & $1.77(0.41-7.57)$ & 0.438 \\
\hline 70 & $4.86(0.64-36.45)$ & 0.124 & $3.22(0.76-13.67)$ & 0.112 \\
\hline \multicolumn{5}{|c|}{ FIGO stage: } \\
\hline I & Reference category & & Reference category & \\
\hline II & $0.99(0.36-2.73)$ & 0.996 & $0.71(0.31-1.64)$ & 0.430 \\
\hline III & $2.20(0.82-5.89)$ & 0.116 & $1.84(0.83-4.09)$ & 0.130 \\
\hline \multicolumn{5}{|l|}{ Grade: } \\
\hline 1 & Reference category & & Reference category & \\
\hline 2 & $1.51(0.71-3.19)$ & 0.282 & $1.77(0.94-3.33)$ & 0.078 \\
\hline 3 & $3.37(1.52-7.44)$ & 0.003 & $2.63(1.27-5.43)$ & 0.009 \\
\hline
\end{tabular}




\section{Cumulative percentages of local relapse according to differentiation}

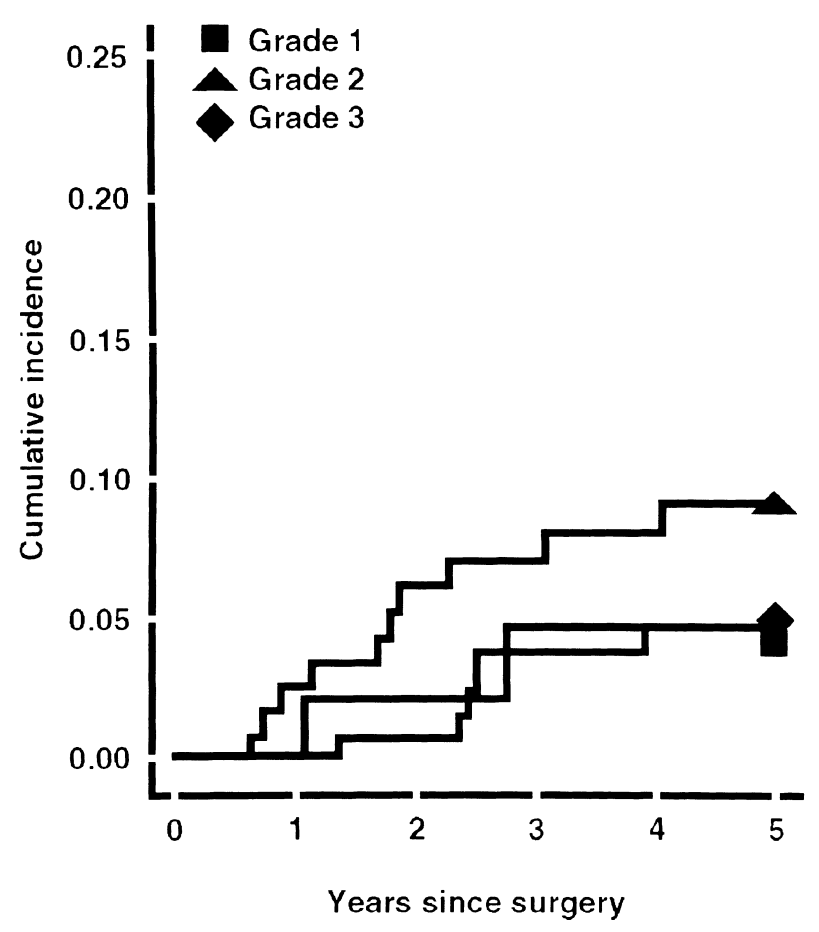

Fig. 1. Cumulative percentages of local relapse according to differentiation.

\section{Cumulative percentages of metastases according to differentiation}

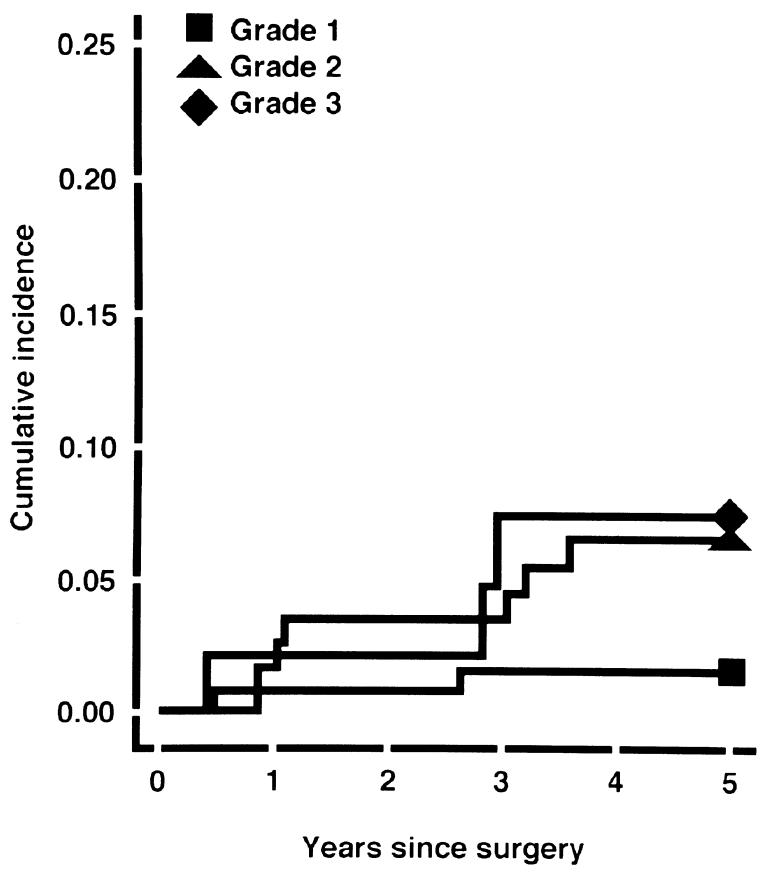

Fig. 2. Cumulative percentages of metastases according to differentiation.

\section{Cumulative percentages of death according to differentiation}

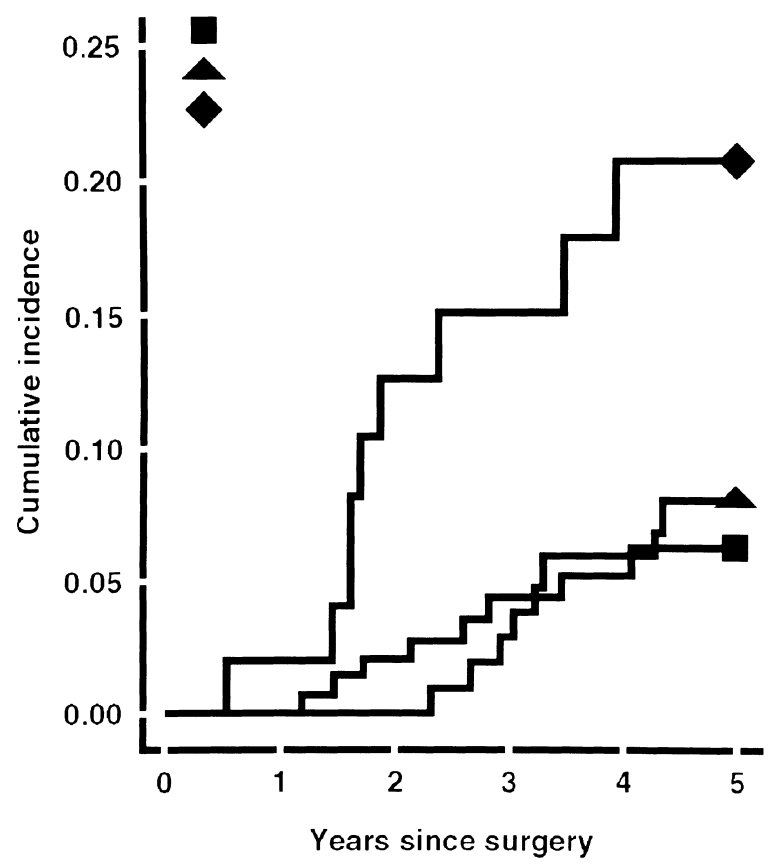

Fig. 3. Cumulative percentages of death according to differentiation.

gressed at a relatively slow pace, possibly allowing for the detection of local relapse and metastases. On the other hand G3 patients, in whom a high mortality has been observed as opposed to a low rate of local or distant metastases, were probably prone to a rather fast disease progression, in which only the final stage of the evolution, death, was unfortunately detected.

Factors associated with the high grade histological variant that may have a bearing on prognosis include a tendency for higher mean age at diagnosis, a greater incidence of both deep myometrial invasion and lymph node involvement [3], and a high risk of clinical understaging as well as a higher rate of vaginal recurrences [4]. Age at diagnosis did not appear as a significant prognostic factor, though for both OS and DFS the estimated HRs showed a trend in the expected direction. This contrasts with other papers showing that age has a prognostic impact $[18,23]$. Our small number of events might not have allowed us to detect this effect, possibly of small magnitude. One might not exclude a bias in the results due to competing causes of death especially acting in older age groups. Our observations cover a time span of over 15 years during which cause of death attribution has not been performed systematically and according to consistent standards. Even if cause of death was available for all patients, we would question its precision and especially so for older patients. Additionally, the focus of the report is on tumor grade, which was not significantly associated 
with age. Since the aim of this contribution was to compare the pattern of occurrence of the events contributing to disease-free survival among tumor grade subgroups, the results observed should at least qualitatively, if not in magnitude, be considered. In particular, the possible bias introduced by death due to other causes than cancer should equally affect all subgroups of grade. Furthermore, the tests comparing the cumulative incidence of either local relapse or metastasis or death among tumor grade subgroups was stratified by stage, which was found to be significantly associated with age. This approach should control the potential bias introduced by causes of death other than cancer possibly occurring more frequently in older age.

Although pelvic and para-aortic lymphadenectomy are now mandatory, especially for grade 3 patients, the procedure was not systematically performed in this series. Only the depth of myometrial invasion was taken into account for prescribing adjuvant radiation therapy. We have not seen an effect of myometrial infiltration on DFS or on OS, which contrasts with previous reports $[2,18-$ $21,23]$. There might be two explanations. On the one hand, it is possible that as the difference in prognosis being relatively small between stage IA, IB and IC, we have not been able to detect it with our sample. On the other hand, therapy was tailored to stage and the two factors may be confounded with each other in such a way to mask their respective effect. The effect of stage was not of specific interest, and our analytic approach (multivariate analysis and stratified testing) has in any case allowed for its role, whether as a prognostic factor or as a confounder.

It is well established that postoperative irradiation reduces the incidence of vaginal recurrences and distant metastases, and improves survival rates in patients with more than one-half myometrial invasion and/or grade 2 or 3 stage I endometrial adenocarcinoma [24-32]. One wonders then if a more aggressive adjuvant radiation therapy (more than $45 \mathrm{~Gy}$ on the pelvic volume or irradiation of the whole abdominal cavity) or a more intensive follow-up of such patients would be justified. A strong evidence is available in favour of the first practice [33]. However the option of a more intensive follow-up would be appropriate only if the efficacy of a second line treatment could be demonstrated.

\section{References}

[1] DiSaia PJ, Creasman WT, Boronow RC, Blessing JA. Risk factors and recurrent patterns in stage I endometrial cancer. Am J Obstet Gynecol 1985;151:1009-15.

[2] Mammoliti S, Bruzzone M, Chiara S et al. Clinical stage I and II endometrial carcinoma: multivariate analysis of prognostic factors. Anticancer Res 1992;12:1415-8.

[3] Creasman WT, Boronow RC, Morrow CP, DiSaia PJ, Blessing J. Adenocarcinoma of the endometrium: its metastatic lymph node potential. Gynecol Oncol 1976;4:239-43.
[4] Price JJ. Vaginal involvement in endometrial carcinoma. Am J Obstet Gynecol 1965;91:1060-5.

[5] Announcement. Changes in gynecologic cancer staging by the International Federation of Gynecology and Obstetrics. Am J Obstet Gynecol 1990;162:610-11.

[6] Aalders J, Abeler V, Kolstad P, Onsrud M. Postoperative external irradiation and prognostic parameters in stage I endometrial carcinoma. Clinical and histopathologic study of 540 patients. Obstet Gynecol 1980;56:419-26.

[7] Ahmad NR, Lanciano R, Corn BW, Schultheiss T. Postoperative radiation therapy for surgically staged endometrial cancer: impact of time factor (overall treatment time and surgery-to-radiation interval) on outcome. Int J Radiat Oncol Biol Phys 1995;33:837-42.

[8] Rush S, Gal D, Potters L, Bosworth J, Lovecchio J. Pelvic control following external beam radiation for surgical stage I endometrial adenocarcinoma. Int J Radiat Oncol Biol Phys 1995;33:851-4.

[9] Stata. Computing Resource Center Reference Manual (1991).

[10] Kaplan EL, Meier P. Nonparametric estimation from incomplete observations. J Am Stat Assoc 1958;53:457-81.

[11] Greenwood M. Reports on public health and medical subjects: the natural duration of cancer. HMSO 1926;33:1-16.

[12] Peto R, Pike MC, Armitage P, Breslow NE, Cox DR, Howard SV, Mantel N, McPherson K, Peto J, Smith PG. Design and analysis of randomized clinical trials requiring prolonged observation of each patient. II. Analysis and examples. Br J Cancer 1977;35:1-39.

[13] Cox DR. Regression models and life tables. J R Stat Soc B 1972;34:187-220.

[14] Kalbfleisch JD, Prentice RL. In: The statistical analysis of failure time data, New York: Wiley, 1980.

[15] Pepe MS, Mori M, Kaplan-Meier EL. Marginal or conditional probability curves in the analysis of competimg risk failure time data. Stat Med 1993;12:737-51.

[16] Piver MS, Vongtama V. Parameters adversely affecting survival in women with stage I endometrial carcinoma. Am J Obstet Gynecol 1965;91:1060-5.

[17] Malkasian GD, Annegers JF, Fountain KS. Carcinoma of the endometrium: Stage I. Am J Obstet Gynecol 1980;136:872-88.

[18] Connelly PJ, Alberhasky RC, Christopherson WM. Carcinoma of the endometrium. III. Analysis of 865 cases with adenocarcinoma and adenoacanthoma. Obstet Gynecol 1982;59:569-75.

[19] Hendrickson M, Ross J, Eifel P, Cox RS, Martinez A, Kempson R. Adenocarcinoma of the endometrium: analysis of 256 cases with carcinoma limited to the uterine corpus. Gynecol Oncol 1982;13:373-92.

[20] Boronow Rc, Morrow CP, Creasman WT, DiSaia PJ, Silverberg SG, Miller A, Blessing JA. Surgical staging in endometrial cancer. I. Clinical-pathologic findings of a prospective study. Obstet Gynecol 1984;63:825-32.

[21] Burke TW, Heller PB, Woodward JE, Davidson SA, Hoskins WJ, Park RC. Treatment failure in endometrial carcinoma. Obstet Gynecol 1990;75:96-101.

[22] Morrow CP, Bundy BN, Kurman RJ, Creasman WT, Heller P, Homesley HD, Graham JE. Relationship between surgicalpathological risk factors and outcome in clinical stage I and II carcinoma of the endometrium: a Gynecologic Oncology Group Study. Gynecol Oncol 1991;40:55-65.

[23] Christopherson WM, Connelly PJ, Alberhasky RC. Carcinoma of the endometrium: an analysis of prognosticators in patients with favorable subtypes and stage I disease. Cancer 1983;51:1705-9.

[24] Vangtoma J, Kurohara S, Badihi AO, Webster JH. The value of adjuvant irradiation in the treatment of endometrial carcinoma Stage I, Group I. Cancer 1970;25:45-9.

[25] Joslin CA, Vaishampayan GV, Mallik A. The treatment of early cancer of the corpus uteri. Br J Radiol 1977;50:38-45.

[26] Reddy S, Lee MS, Henderson FR. Patterns of recurrences in endometrial carcinoma and their management. Radiol 1979;133:737-40. 
[27] Aalders J, Abeler V, Kolstad P, Onsrud M. Postoperative external irradiation and prognostic parameters in stage I endometrial carcinoma. Obstet Gynecol 1980;56:419-26.

[28] Marchetti L, Piver S, Tsukoda Y, Reese P. Prevention of vaginal recurrence of stage I endometrial adenocarcinoma with postoperative vaginal radiation. Obstet Gynecol 1986;67:399-402.

[29] Nori D, Hilaris BS, Tome M, Lewis Jr. JL, Birnbaum S, Fuks Z. Combined surgery and radiation in endometrial carcinoma: an analysis of prognostic factors. Int J Radiat Oncol Biol Phys 1987;13:489-97.

[30] Meerwaldt JH, Hoekstra CJM, van Putten WLJ, Subandono Tjokrowardojo AJ, Koper PCM. Endometrial adenocarcinoma adjuvant radiotherapy tailored to prognostic factors. Int $\mathrm{J}$ Radiat Oncol Biol Phys 1990;18:299-304.
[31] Sorbe BG, Smeds AC. Postoperative vaginal irradiation with high dose rate afterloading technique in endometrial carcinoma stage I. Int J Radiat Oncol Biol Phys 1990;18:305-14.

[32] Elliott P, Green D, Coates A, Krieger M, Russel P, Coppleson M, Solomon J, Tattersall M. The efficacy of postoperative vaginal irradiation in preventing vaginal recurrence in endometrial cancer. Int J Gynecol Cancer 1994;4:84-93.

[33] Gibbons S, Martinez A, Schray M, Podratz K, Stanhope R, Garton G, Weiner S, Brabbins D, Malkasian G. Adjuvant whole abdominopelvic irradiation for high risk endometrial carcinoma. Int $\mathrm{J}$ Radiat Oncol Biol Phys 1991;21:1019-25. 